\title{
Effect of Nonzero Write Field Rise Time in Digital Magnetic Recording
}

\author{
J. FLUITMAN
}

\begin{abstract}
A general method is given to predict write field rise time effects on isolated pulses in digital magnetic recording. The method is based on the use of a graph, which can be calculated once and for all, from which the position and the form of a transition, written under several conditions, can be derived. Emphasis is laid on pulse shift effects, and it is shown that the results obtained give good estimates, in spite of the approximations used.
\end{abstract}

\section{INTRODUCTION}

Although a considerable amount of literature has been devoted to the digital recording process, only minor attention has been given to explicit treatments of write field rise time effects. One treatment has been given by Lee and Truman [1], along the lines suggested by Ku [2], but they only consider the case of writing on a medium which is initially remanent at $-M_{r}$ and the head field switching from negative to positive. Other investigations consider nonzero write field rise times at best in an implicit way, in computer simulations of the recording write process [3], [4]. From these treatments it cannot become clear what exactly is the contribution of the nonzero rise time to the results.

It is the purpose of the present work to develop a method by which rise time effects can be studied explicitly in their dependence on the magnetic properties of the medium, the initial remanent state of the medium, the velocity of the medium relative to the head, etc. An important assumption in our work (which will be discussed later on) is the absence of recording demagnetization. This approximation is necessary to make the problem tractable, although it can be expected that recording demagnetization will interfere with zise time effects. On the other hand, a separate study of pulse shift and pulse widening, caused by nonzero rise times, can give an insight into the conditions under which such effects can become dominant. For the sake of clarity we will concentrate on pulse shift effects. It will be clear at the end that pulse widening effects can be treated correspondingly.

A general outline of the problem can be given with the help of Figs. 1, 2, and 3. In Fig. 1 the recording head gap region, together with the recording medium is shown schematically. The gap length is $2 g$, the head/medium separation is $y$. The medium moves with a velocity $v$ relative to the head in the positive $x$-direction. We will use a coordinate $x_{s}$, fixed to the head,

Manuscript received March 3, 1975; revised November 10, 1975.

The author is with the Department of Electrical Engineering, Twente University of Technology, Enschede, The Netherlands. and a coordinate $x_{m}$, fixed to the medium, in such a way that $x_{m}=x_{s}-v t$.

Fig. 2 shows the write field, switching linearly from negative to positive. Contrary to convention the positive direction of the time axis has been drawn from right to left (which proved to be convenient in drawing this illustration). The same holds for the $x_{s}$ axis. A particle of the layer, say $x_{m_{1}}$, will travel along the head gap region, obeying $x_{m_{1}}=x_{s}-v_{1} t\left(v=v_{1}\right.$ for this example), which can be represented by a straight line in the ground plane of the diagram. It is easy now to get an impression of the magnetic fields experienced by the particle $x_{m}$, during the passage. In general, a period of negative fields is followed by a period of positive fields and, as illustrated in Fig. 3, the extreme field values $H_{\min }$ and $H_{\max }$ are sufficient to determine the final state of remanence of the particle, once the initial state is given. In this way the final state of rema-

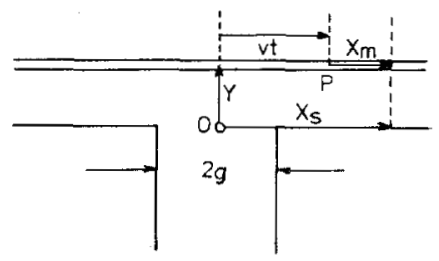

Fig. 1. Recording head gap region.

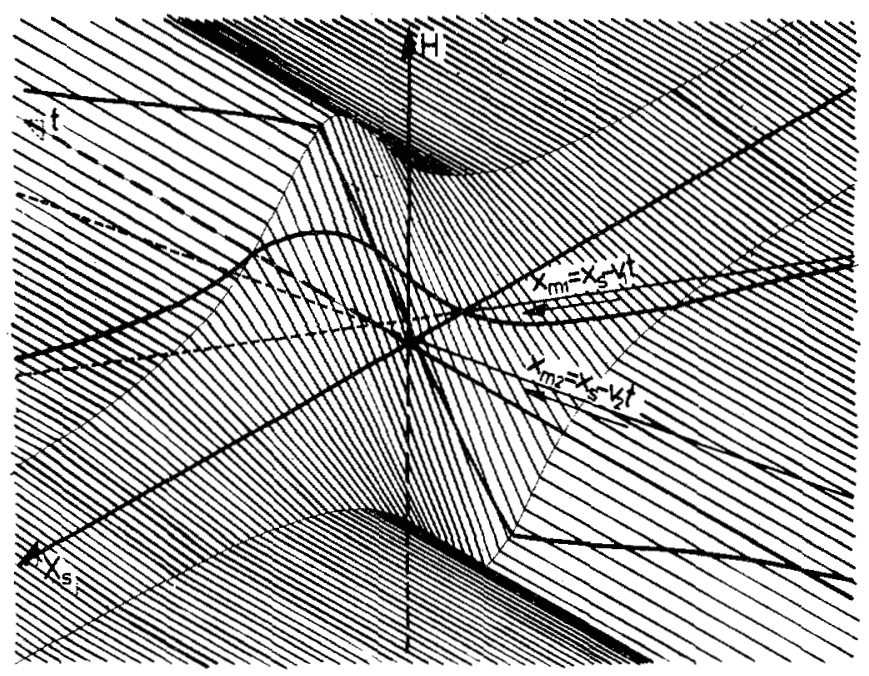

Fig. 2. Head field as a function of $x_{S}$ and $t$ during a linear transition. The positive direction of both the $x_{s}$ and $t$ axes point to the left. Examples of "field histories" are given for a particle with coordinate $x_{m_{1}}$ moving with a velocity $v_{1}$ and a particle with coordinate $x_{m}$ moving with a velocity $v_{2}$ relative to the head. $v_{1}$ and $v_{2}$ can be interpreted as real or as reduced velocities (see text). 


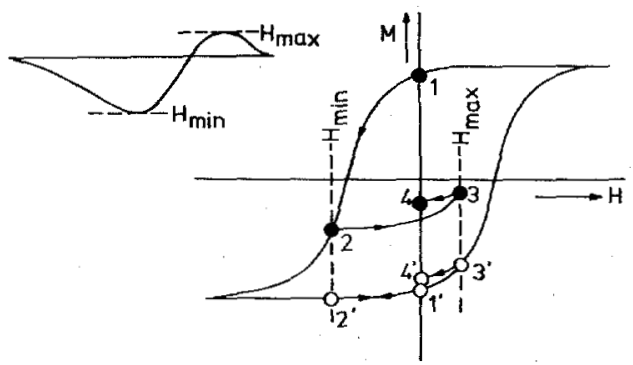

Fig. 3. A field history, as depicted in the inset, can lead to different final states of remanence depending on the initial states (trajectories $1-2-3-4$ and $\left.1^{\prime}-2^{\prime}-3^{\prime}-4^{\prime}\right)$.

nence of all points of the medium can be tracked and the transition can be constructed. It is clear that the initial state of remanence is of great importance and nonlinear pulse crowding effects may result when information is written in a randomly premagnetized medium.

\section{STARTING POINTS}

We will first summarize the basic assumptions underlying our analysis, and call attention to a few points which can be of help later on.

1) Magnetization of the medium only occurs along the $x$-direction. Only the $x$-component of the head field is considered.

2) The layer thickness is small enough to guarantee that the head field is uniform over the thickness.

3) The effect of recording demagnetization will be ignored.

4) Initially the head field will have the form

$$
H\left(x_{s}\right)=\frac{C}{x_{1 / 2}^{2}+x_{s}^{2}}
$$

where $x_{s}$ is the coordinate relative to the head (Fig. 1), $x_{1 / 2}$ is half the $50 \%$ width of the head field, and $C$ is a constant of proportionality. The maximum head field $H_{\text {top }}$ is found for $x_{s}=0$

$$
H_{\text {top }}=\frac{C}{x_{1 / 2}^{2}} \text {. }
$$

5) As a first approximation the time dependence of the head field will be assumed linear during switching. If the time dependent head field is denoted as $H^{*}\left(x_{s}, t\right)$, then we have $H^{*}\left(x_{s}, t\right)=H\left(x_{s}\right) f(t)$ with

$$
\begin{aligned}
& f(t)=-1, \quad \text { for } t<-T / 2 \\
& f(t)=t / T, \quad \text { for }-T / 2 \leqslant t \leqslant T / 2 \\
& f(t)=1, \quad \text { for } t>T / 2 .
\end{aligned}
$$

The switching time is $T$.

6) Besides an $x$-coordinate, fixed to the recording head and denoted by $x_{s}$, we consider an $x$-coordinate fixed to the medium, denoted by $x_{m}$, in such a way that

$$
x_{m}=x_{s}-v t
$$

where $v$ is the velocity of the medium relative to the head.
7) In the following we take all field strengths normalized to the value of $H_{\text {top }}$, all $x$-coordinate values normalized to $x_{1 / 2}$, and all time valies normalized to $T / 2$. Equations (1), (2), and $(3)$ then transform into

$$
\begin{aligned}
& H\left(x_{s}\right)=\frac{1}{1+x_{s}^{2}} \\
& f(t)=-1, \quad \text { for } t<-1 \\
& f(t)=t, \quad \text { for }-1<t<1 \\
& f(t)=1, \quad \text { for } t>1 \\
& x_{m}=x_{s}-\alpha t
\end{aligned}
$$

where $\alpha=v T /\left(2 x_{1 / 2}\right)$ can be regarded as the normalized medium/head velocity. With these normalized quantities in mind we can look to Fig. 2 in a quite different way. The write field transition now has a fixed universal form, independent of the rise time and the head field parameters. Instead, a rise time variation can be experienced as a variation of the normalized medium/head velocity $\alpha$. In fact, we could have suggested right from the beginning to study rise time effects by taking a standard head field reversal and vary the medium/head velocity $v$.

8) The polarity of the head field reversal is chosen to be from negative to positive. With this polarity any particle of the medium experiences a period of negative fields followed by a period of positive fields. The peak values of the fields will be denoted by $H_{\min }$ and $H_{\max }$ and will be experienced by the particle $x_{m}$ in this order.

9) The final state of remanence of each particle of the medium is completely determined by $H_{\min }$ and $H_{\max }$ and the initial state of remanence of that particle. In our derivation we focus on two cases, namely, that before writing all of the medium is in a state of negative remanence $-M_{r}$, or in a state of positive remanence $+M_{r}$.

10) The position of a transition will be defined by the coordinate $x_{m}$ of the particle which is left in a remanent state $M=0$. Such a particle has a characteristic track in the hysteresis loop (Fig. 3) ending exactly in the origin $(M=0)$.

11) The pulse shift $x_{d}$ will be defined as the position of the transition relative to the position of the transition, written with $T=0$ at $t=0$, which has the coordinate $x_{m}=\sqrt{H_{r c}^{-1}-1}$, so we have

$$
x_{d}=x_{m}(M=0)-\sqrt{H_{r c}^{-1}-1}
$$

$H_{r c}$ is the remanence coercive force.

12) If the initial state of remanence of the medium is $-M_{r}$, then the medium particle which has experienced $H_{\max }=H_{r c}$ will determine the pulse shift, independent of the magnitude of $H_{\min }$. This case has been considered by Lee and Truman [1].

13) If the initial state of remanence of the medium is $+M_{r}$, then there will be a set of $\left(H_{\min }, H_{\max }\right)$ combinations which lead to a final remanence $M=0$. The functional relationship which exists between $H_{\min }$ and $H_{\max }$ will be denoted implicitly by $R\left(H_{\min }, H_{\max }\right)=0$. The functional form of $R$ is dependent on the hysteresis characteristics and can be deter- 
mined from experimentally taken hysteresis loops or derived from a suitable model. The particle of the layer which experiences an $H_{\min }$ and an $H_{\max }$ in sequence, leading to $R=0$, determines the pulse shift in this case.

\section{ANALYTICAL APPROACH}

$H_{\min }$ and $H_{\max }$ are determined by $\left(\partial H^{*} / \partial t\right)_{x_{m}}=0$. Since $H^{*}=H\left(x_{s}\right) f(t)$ and $x_{m}=x_{s}-\alpha t$ it follows:

$$
\alpha \frac{1}{H} \frac{d H}{d x_{s}}=-\frac{1}{f} \frac{d f}{d t}
$$

which can be solved after introduction of $\left(1^{\prime}\right)$ and $\left(2^{\prime}\right)$ and using $\left(3^{\prime}\right)$. The calculation then, is somewhat complicated by the fact that it can only be carried out in parts since the function $f$ is differentiable only in parts. However the calculation is straight forward and leads to the following results, valid for $\alpha \geqslant 1$ : that $R=0$ can be described with the help of a set of linear expressions:

$$
\begin{array}{ll}
H_{\max }-H_{c}=0, & \text { when } H_{\min }<-H_{s} \\
H_{c} H_{\min }+H_{s} H_{\max }=0, & \text { when }\left\{\begin{array}{l}
-H_{s}<H_{\min }<-H_{c} \\
H_{c}^{2} /_{H_{s}}<H_{\max }<H_{c}
\end{array}\right. \\
H_{\min }+H_{c}=0, & \text { when } 0<H_{\max }<H_{c}^{2} l_{H_{s}} .
\end{array}
$$

In this model $H_{r c}=H_{c}$ and a parameter $H_{s}$, the saturation field, has been introduced. The elaboration of this case, that is the calculation from (6) and (7) of the particle coordinate $x_{m}$ satisfying (9) for any value of $\alpha$, is rather lengthy and complicated by the fact that (9) is, again, given in the form of piecemeal differentiable expressions. A general representation of the solution, in a form comparable to (8), will be omitted since this appears to be a lengthy and nontransparant enumera-

$$
\begin{array}{cll}
x_{m}<-\alpha & ; H_{\min }=-1 /\left(1+\left(x_{m}-\alpha\right)^{2}\right) & ; H_{\max }=1 \\
-\alpha<x_{m}<-\sqrt{\alpha^{2}-1} ; H_{\min }=-1 /\left(1+\left(x_{m}-\alpha\right)^{2}\right) & ; H_{\max }=1 /\left(1+\left(x_{m}+\alpha\right)^{2}\right) \\
-\sqrt{\alpha^{2}-1}<x_{m}<\sqrt{\alpha^{2}-1} & ; H_{\min }=\left(-\sqrt{x_{m}^{2}+1}-x_{m}\right) / 2 \alpha ; H_{\max }=\left(\sqrt{x_{m}^{2}+1}-x_{m}\right) / 2 \alpha \\
\sqrt{\alpha^{2}-1}<x_{m}<\alpha & ; H_{\min }=-1 /\left(1+\left(x_{m}-\alpha\right)^{2}\right) & ; H_{\max }=1 /\left(1+\left(x_{m}+\alpha\right)^{2}\right) \\
\alpha<x_{m} & ; H_{\min }=-1 & ; H_{\max }=1 /\left(1+\left(x_{m}+\alpha\right)^{2}\right)
\end{array}
$$

and, for $\alpha<1$ :

$$
\begin{array}{lll}
x_{m}<-\alpha & ; H_{\min }=-1 /\left(1+\left(x_{m}-\alpha\right)^{2}\right) & ; H_{\max }=1 \\
-\alpha<x_{m}<\alpha & ; H_{\min }=-1 /\left(1+\left(x_{m}-\alpha\right)^{2}\right) & ; H_{\max }=1 /\left(1+\left(x_{m}+\alpha\right)^{2}\right) \\
\alpha<x_{m} & ; H_{\min }=-1 & ; H_{\max }=1 /\left(1+\left(x_{m}+\alpha\right)^{2}\right) .
\end{array}
$$

Note, that $\alpha\left(=v T / 2 x_{1 / 2}\right)$ is directly proportional to the rise time $T$, so that we have expressed $H_{\min }$ and $H_{\max }$ in terms of $x_{m}$ and $T$.

The pulse shift can be calculated as a function of $\alpha$ (thus of $T$ ) for the case of initial remanence $-M_{r}$. Therefore, the value of $x_{m}$ must be calculated from $H_{\max }=H_{r c}$, given the value of $\alpha$. Using expressions (6) and (7) the calculation, again, is straightforward and leads to the following formulae:

$$
\begin{aligned}
x_{d}= & \frac{1}{4 \alpha H_{r c}}-\alpha H_{r c}-\sqrt{\frac{1}{H_{r c}}-1}, \\
& \text { when } \frac{1}{2}\left(1-\sqrt{1-\alpha^{-2}}\right)<H_{r c}<\frac{1}{2}\left(1+\sqrt{1-\alpha^{-2}}\right) \\
& \text { which only has a meaning for } \alpha>1 \\
x_{d}= & -\alpha, \quad \text { in all other cases. }
\end{aligned}
$$

We have depicted two characteristic cases in Fig. 4 (b). Note that in the limit of large rise times $x_{d} \approx-\alpha H_{r c}$ (or $x_{d} \approx$ $-(v T / 2)\left(H_{r c} / H_{\text {top }}\right)$, in nonreduced quantities), which can also be derived directly from simple arguments.

The second case, with initial remanence $+M_{r}$, can only be treated if $R\left(H_{\min }, H_{\max }\right)=0$ is given in an analytical form, which, in addition, must be simple enough to lead to solvable equations. This requirement has lead us to the hysteresis model of Fig. 4(a), which has been constructed in such a way tion of expressions and conditions. Instead we give, as an example, a characteristic result (for the case $H_{\text {top }}=H_{s}=2 H_{c}$ ):

$$
\begin{array}{ll}
x_{d}=-\alpha, & \text { when } \alpha<\frac{1}{2} \\
x_{d}=-1+3 \alpha-\sqrt{8 \alpha^{2}-1}, & \text { when } \frac{1}{2}<\alpha<\frac{3}{4} \sqrt{ } 2 \\
x_{d}=-1+\frac{1}{4} \sqrt{ } 2, & \text { when } \frac{3}{4} \sqrt{ } 2<\alpha<\sqrt{ } 2 \\
x_{d}=-1+\frac{1}{2} \alpha-\frac{1}{2 \alpha}, & \text { when } \sqrt{ } 2<\alpha .
\end{array}
$$

Fig. 4. (a) Hysteresis model, in which the minor loops are composed of parallel straight lines bending at the dotted line which connects the lower right corner of the major loop with the point with coordinate $H_{c}^{2} / H_{\text {s }}$ on the $H$ axis. This model leads to expression (9) in the text. (b) Pulse shift/rise time curves derived analytically for the case of initial negative remanence (denoted by - ) and initial positive remanence (denoted by + ) for two values of $H_{c} /_{H_{\text {top }}}$. Use is made of the hysteresis model of Fig. 4(a) with the condition $H_{s}=2 H_{C}$ $\left(H_{c}=H_{r c}\right)$. 
In Fig. $4(b)$ we have depicted this result (together with the case $H_{\text {top }}=3 /_{2} H_{s}=3 H_{c}$ ). It can be shown that, in the limit of large rise times, the results approach $x_{d} \approx \alpha H_{r c}$, in agreement with expectation.

\section{GRAPHICAL APPROACH}

Since $H_{\min }$ and $H_{\max }$ both are functions of $x_{m}$ and $\alpha$, we can make a diagram of $H_{\min }$ against $H_{\max }$ and represent any particle $x_{m}$, at any value of $\alpha$, by a dot in the diagram. In this way we can fill the diagram as has been done in Fig. 5. As a matter of fact, we have calculated $H_{\min }, H_{\max }$ values for fixed values of $x_{m}$ letting $\alpha$ vary within a certain range and, on the other hand, for fixed values of $\alpha$, letting $x_{m}$ vary. In this way iso- $x_{m}$ curves (upper left to lower right in the diagram) and iso- $\alpha$ curves (lower left to upper right) can be found. The iso- $\alpha$ curves can be symbolized by means of implicit functional relations $S_{\alpha}\left(H_{\min }, H_{\max }\right)=0$. The form of $S$ depends on the value of $\alpha$ and the relation describes the set of $H_{\min }, H_{\max }$ values which, together, form an iso- $\alpha$ curve in the diagram. The derivation of the pulse shift/rise time behavior now proceeds as follows.

1) For the initial state $-M_{r}$, a particle $x_{m}$ has to be found, which has experienced $H_{\max }=H_{r c}$, for any given value of $\alpha$. This means that the intersection of the curve $S_{\alpha}\left(H_{\min }, H_{\max }\right)=0$, for any given value of $\alpha$, with the straight line $H_{\max }=H_{r c}$ must be read from the diagram. For instance, if $H_{r c}=0.45$ (straight line in Fig. 5) and $\alpha=0.9$ a value of $x_{m} \approx 0.18$ is found (after interpolation between the two $x_{m}$ values which are nearest by).

2) For the initial state $+M_{r}$ the procedure is exactly the same, now the intersection of $S_{\alpha}\left(H_{\min }, H_{\max }\right)$ with the curve $R\left(H_{\min }, H_{\max }\right)=0$ has to be found. In Fig. 5 we have drawn the curve $R=0$, which has been derived from the hysteresis loop of an unoriented $\gamma \mathrm{Fe}_{2} \mathrm{O}_{3}$ layer.

The results of a graphical analysis for this medium is presented in Fig. 6 (drawn curves) together with, among others, the result of our analytical solution (with $H_{c}=0.5$ in reduced form, comparable to $H_{r c}=0.45$ for the $\gamma \mathrm{Fe}_{2} \mathrm{O}_{3}$ example). As can be seen, both results are in close agreement although the hysteresis characteristics are quite different for the two cases.

A great advantage of our method is that use can be made of a single graph which has been calculated once and for all, which can be used for a number of applications. The procedure works for any hysteresis form and for any initial and final state of remanence. Therefore not only pulse shifts can be derived in this way, but also pulse widths and even complete pulse forms, if one wishes. To derive the pulsewidth for in-

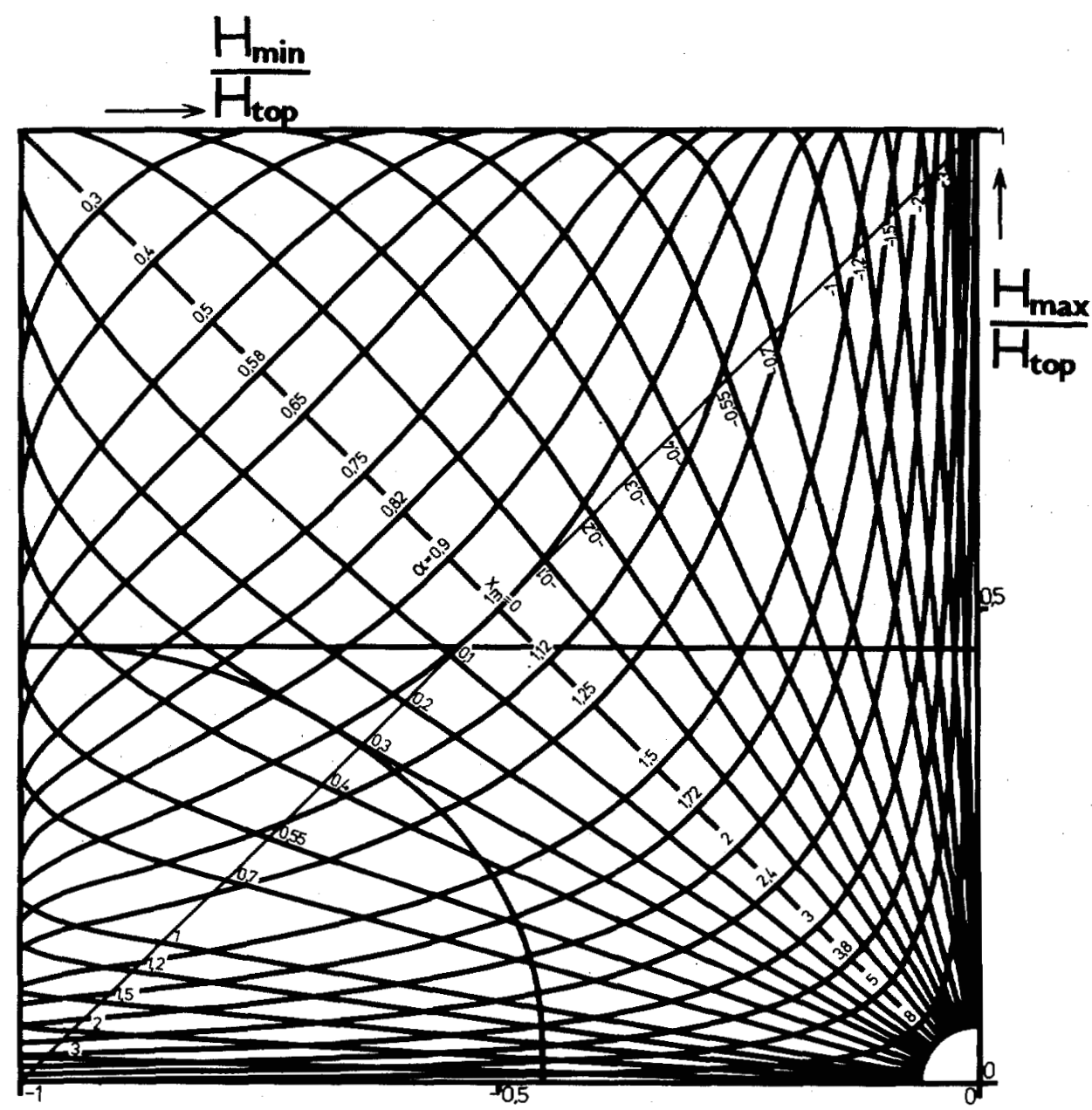

Fig. 5. Diagram, from which can be read for any particle $x_{m}$, at any value of $\alpha$, the values of $H_{\min } / H_{\text {top }}$ and $H_{\text {max }} / H_{\text {top }}$ The diagram is a graphical representation of the results (6) and (7). "Characteristic curves" $H_{\max }=H_{r c}$ (reduced quantities $)$ and $R\left(H_{\min }, H_{\max }\right)=0$ are drawn for a typical case of recording in an unoriented $\gamma \mathrm{Fe}_{2} \mathrm{O}_{3}$ layer. 


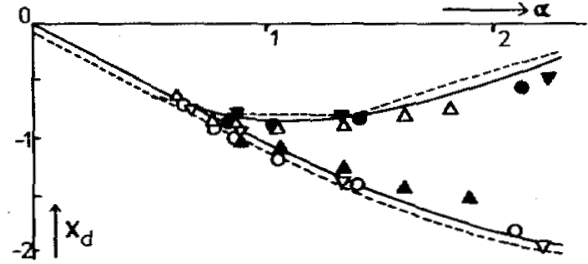

Fig. 6. Pulse shift/xise time curves resulting from a graphical analysis for a typical case of recording in an unoriented $\gamma \mathrm{Fe}_{2} \mathrm{O}_{3}$ layer (drawn curves). The circles and triangles are the results of computations using the Karlquist expression (10) and putting $x_{1 / 2}=\sqrt{y^{2}+g^{2}}(\Delta, \Delta$; $y / g=0.5, \bullet: y / g=1, \nabla: y / g=2)$. The dashed curve is the analytical result already presented in Fig. $4(\mathrm{~b})\left(H_{c} / H_{\text {top }}=0.5\right.$, the curve is shifted a little bit for clarity). The upper curve is for initial remanence $+M_{r}$, the lower curve for $-M_{r}$.

stance, one must look for those particles $x_{m}$ which end in a final state of remanence $M=+M_{\mathrm{p} . \mathrm{w}}$, and $M=-M_{\mathrm{p} \text {.w. }}, M_{\mathrm{p} . \mathrm{w}}$. being short for the remanent state which one has selected as characteristic for the pulsewidth, for instance $M_{\mathrm{p} . w}$. might be $\frac{1}{2} M_{r}$. Now one must calculate or measure "characteristic curves" (like $H_{\max }=H_{r c}$ or $R\left(H_{\min }, H_{\max }\right)=0$ ) which are representative for those sets of $\left(H_{\min }, H_{\max }\right)$ combinations which lead to final states of remanence $+M_{\mathrm{p} . \mathrm{w}}$, and $-M_{\mathrm{p} . \mathrm{w}}$, respectively, starting from initial states of $+M_{r}$ and $-M_{r}$. The characteristic functions can be drawn in the graph and for any $\alpha$, and for both $+M_{r}$ and $-M_{r}$ as starting values, one can find a particle $x_{m_{+}}$which ends at $+M_{\text {p.w. }}$ and $x_{m_{-}}$which ends at $-M_{\text {p.w. }} .\left|x_{m_{+}}-x_{m_{-}}\right|$then is the pulsewidth.

A little complication of our method seems to be that the procedure only works at the interior of the diagram and not at the edges, when $H_{\min }=-1$ and $H_{\max }=H_{r c}$ for instance (the starting point of the "characteristic curves" in Fig. 5). A single $\left(H_{\min }, H_{\max }\right)$ pair on the left edge (as is also true for the upper edge with $H_{\max }=1$ ) is not generated by a single pair of $\left(x_{m}, \alpha\right)$ values but by a set of $\left(x_{m}, \alpha\right)$ pairs instead. And, of course these pairs, which ultimately give a part of the pulse shift/rise time curve, cannot be read from the diagram, hidden as they are under the point with $H_{\min }=-1$. However, formulae (6) and (7) tell that for $H_{\min }=-1, H_{\max }=1 /(1+$ $\left.\left(x_{m}+\alpha\right)^{2}\right)$ so that when $H_{\max }$ is taken equal to $H_{r c}$, the relation between $x_{m}$ and $\alpha$ is determined by

$$
\frac{1}{1+\left(x_{m}+\alpha\right)^{2}}=H_{r c} \text {. }
$$

This gives $x_{m}+\alpha=\sqrt{H_{r c}^{-1}-1}$ and thus $x_{d}=-\alpha$. So we can conclude that all pulse shift/rise time curves start likewise as $x_{d}=-\alpha$, for both the initial state of remanence $+M_{r}$ and $-M_{r}$. Also it can be seen from (7) that the point of divergence of the drawn curves for initial remanence $+M_{r}$ and $-M_{r}$, in Fig. 6, is determined by the boundary condition $x_{m}=\alpha$ for which $H_{\min }=-1$ stops to be valid. Introducing $x_{m}=\alpha$ into $x_{m}+\alpha=$ $\sqrt{H_{r c}^{-1}-1}$, we find $\alpha_{b}=\frac{1}{2} \sqrt{H_{r c}^{-1}-1}$ as the boundary value for $\alpha$, so for $\alpha<\alpha_{b} x_{d}=-\alpha$ for any initial remanent state. In some cases $\alpha_{b}$ may be larger than $\frac{1}{2} \sqrt{H_{r c}^{-1}-1}$. If the line $H_{\max }=H_{r c}$ and the curve $R=0$ do not diverge right at the starting point at the left edge ((9) provides an example), but coincide, partly, at the interior of the diagram, then $\alpha_{b}$ is equal

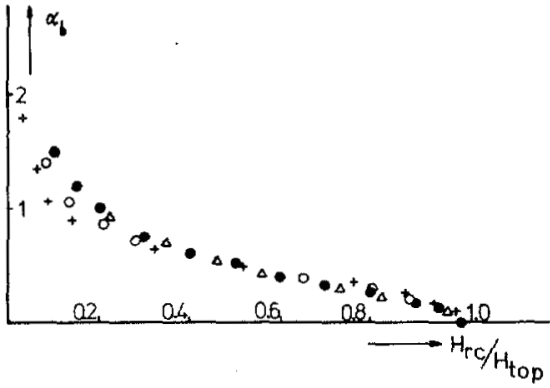

Fig. 7. $\alpha_{b}$ as a function of $H_{r c} / H_{\text {top }} \cdot \alpha_{b}$ is the boundary value of $\alpha$, below which the pulseshift does, in general, not depend on the initial remanence. Filled circles are analy tic results. Open triangles, open circles and crosses are results calculated with the help of the Karlquist expression $(10)(y / g=2,1$, and 0.5 , respectively).

to the value of $\alpha$ under the point of divergence of $H_{\max }=H_{r c}$ and $R=0$ in the diagram.

The relation $\alpha_{b}=\frac{1}{2} \sqrt{H_{r c}^{-1}-1}$ is depicted in Fig. 7. As can be seen $\alpha_{b}=0$ for $H_{r c}=1$. This is a worst case situation with respect to pulse shift effects. $H_{r c}=1$, expressed in nonreduced quantities, means $H_{\text {top }}=H_{r c}$ (which can only make sense for a square hysteresis loop), and reflects a rather extreme situation. In practice $H_{r c} \approx 0.5$ giving $\alpha_{b} \approx 0.5$.

\section{COMMENTS AND CONCLUSIONS}

The convenience of the graphical method relies for the greater part on the possibility to express the time dependent head field in terms of the reduced quantities $x_{s}$ and $t$ with no other parameters left in the expression $\left(1^{\prime}\right),\left(2^{\prime}\right)$. This is no longer possible when the Karlquist expression

$$
\frac{H}{H_{\text {top }}}=\frac{\tan ^{-1}\left(g+x_{s}\right) / y+\tan ^{-1}\left(g-x_{s}\right) / y}{2 \tan ^{-1} g / y}
$$

is used for the head field. In this case the quantities $x_{s}$ and $y$ cannot be reduced in such a way that only a reduced quantity $x_{s}$ remains. (In expression (1) the dependence of the head field on $y$ is incorporated in the supposed dependence of $x_{1 / 2}$ on $y$.) If one wants to follow the procedure outlined in the preceding section, one would be forced to calculate graphs like that of Fig. 5 for any value of $y$ which is under consideration, and this seems not to be very attractive.

On the other hand, the significance of results obtained, with a head field in the simple form (1) depends on the accuracy with which results obtained with a head field in the form (10), for example, can be approached. We have examined this by recalculating the results of Figs. 6 and 7, starting from the expression (10) instead of (1), for a few characteristic cases $(y / g=$ $0.5,1.0$, and 2 , respectively). Thereby we have approximated the half $50 \%$ width of the Karlquist field by $x_{1 / 2}=\sqrt{y^{2}+g^{2}}$. This choice is acceptable for both $y \gg g$ and $y<<_{g}$ (contrary to the "choice" of $x_{1 / 2}=\sqrt{y^{2}-g^{2}}$, which is suggested by the form of (10) in the limiting case $y \gg g$, see [1] formulas (3) and (4)). As can be seen from the figures the results for the simple field form (1) are in good agreeneent with those for the Karlquist field and as far as there are deviations they tend to reduce the rise time effects. The latter is obviously due to the fact that our approximating head field (1) underestimates the 
field strength values in the most important region (50\% width region).

Our conclusion is that rise time problems can be approached to a good degree of accuracy with the help of diagram 5 and, without further proof, we believe this to be true also for head fields other than (10) (finite pole tip head fields, for instance) if a proper choice is made for $x_{1 / 2}$.

If an exponential rise time is considered instead of a linear one, diagram 5 is no longer valid and has to be recalculated. This again can be done once and for all, since an exponential rise time can also be given in a simple reduced form. We have not worked out this case, but the procedure is exactly the same.

It is difficult to make a direct estimate of the accuracy of our results with respect to recording demagnetization. Therefore we have compared the results of Fig. 6 with results obtained from a dynamic self-consistent computer simulation of the write process (see, for example, [4]). Typical results are depicted in Fig. 8. As can be seen, recording demagnetization on the scale of our calculations (valid for "thick" $\gamma \mathrm{Fe}_{2} \mathrm{O}_{3}$ layers) seems to affect our results only in an additive way, which means that the mutual accuracy is not affected seriously.

As an example we may choose $\alpha_{b} \approx 0.5$ (for $H_{r c} \approx 0.5$, see Fig. 7). In this case $T_{b} \approx x_{1 / 2} / v$, in nonreduced quantities, where $T_{b}$ is the critical value of $T$, below which the pulse shift does not depend on the initial state of remanence. If $x_{1 / 2}=2.5 \mu \mathrm{m}, v=50 \mathrm{~m} / \mathrm{sec}$, for example, it follows that $T_{b} \approx$ $50 \mathrm{nsec}$. (Note that pulse shift effects can be reduced by an increase of $x_{1 / 2}$.)

Pulse widening can be studied along the same lines following the procedure outlined in the preceding section. A general property of the pulse widening/rise time behavior is that the pulsewidth does not depend on the rise time for values of $\alpha \lesssim 1$ and increases with increasing values of $\alpha \gtrsim 1$. The increase is about $10 \%$ for $\alpha=2$ for the case of a unoriented $\gamma \mathrm{Fe}_{2} \mathrm{O}_{3}$ layer. Recording demagnetization reduces this percen-

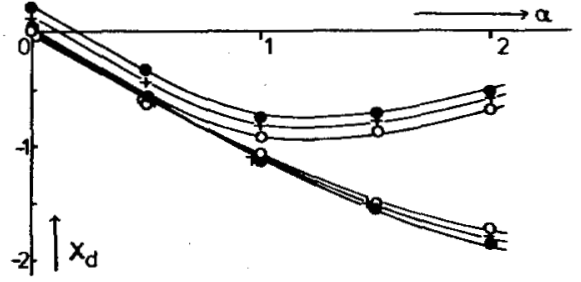

Fig. 8. Results for the pulse shift/rise time behavior as computed with a dynamic self-consistent computer simulation. Results are for unoriented $\gamma \mathrm{Fe}_{2} \mathrm{O}_{3}$ layers with thickness $0 \mu \mathrm{m}(0), 1 \mu \mathrm{m}(\mathrm{x})$, and $2 \mu \mathrm{m}(\bullet)$.

tual increase. For small values of $\alpha(\alpha \lesssim 1)$ the pulseform depends largely on the contour of the head field which is different for the fields (1) and (10).

Our final conclusion is that the analysis of rise time effects along the lines presented in the preceding pargraphs lead to accurate estimates and can be used to predict the circumstances under which such effects may occur. In general, rise time effects can be avoided if it is possible to choose $T<T_{b}$, with $T_{b}=\left(2 x_{1 / 2} / v\right) \alpha_{b}$.

\section{ACKNOWLEDGMENT}

We like to thank. Mr. H. Jansen for his contribution in programming the recording simulation leading to Fig. 8.

\section{REFERENCES}

[1] J. E. Lee and N. N. Truman, "The Effect of Finite Flux Rise Time on Recording Performance," IEEE Trans. Mag. MAG.6, 95-100 (1970).

[2] T. C. Ku, "An Analytical Expression for Describing the Write Process in Magnetic Recording," Prac. IRE, p. 1337, Aug. 1961.

[3] J. R. Herbert and D. W. Patterson, "A Computer Simulation of the Magnetic Recording Process," IEEE Trans. Mag. MAG-1, 352357 (1965).

[4] R. I. Potter and R. J. Schmulian, "Self-Consistently Computed Magnetization Patterns in Thin Magnetic Recording Media," IEEE Trans. Mag. MAG-7, 873-880 (1971). 